\title{
POROSITY, $\sigma$-POROSITY AND MEASURES
}

\author{
M. E. MERA, M. MORÁN, D. PREISS, AND L. ZAJÍČEK
}

\begin{abstract}
We show that given a $\sigma$-finite Borel regular measure $\mu$ in a metric space $X$, every $\sigma$-porous subset of $X$ of finite measure can be approximated by strongly porous sets. It follows that every $\sigma$-porous set is the union of a $\sigma$-strongly porous set and a $\mu$-null set. This answers in the positive the question whether a measure which is absolutely continuous with respect to the $\sigma$-ideal of all $\sigma$-strongly porous sets is absolutely continuous with respect to the $\sigma$-ideal of all $\sigma$-porous sets. Using these results, we obtain a natural decomposition of measures according to their upper porosity and obtain detailed information on values that upper porosity may attain almost everywhere.
\end{abstract}

\section{IntRoduction AND NOTES ON THE NOTIONS OF POROSITY}

We show that from the point of view of $\sigma$-finite Borel regular measures (on separable metric spaces) the difference between porous and strongly porous sets is negligible in a rather strong sense: $\sigma$-porous sets of finite measure become strongly porous after deleting sets of arbitrarily small measure and all $\sigma$-porous sets become $\sigma$-strongly porous after deleting a null set. As a corollary we obtain a negative answer to Question 4.31 of [8] whether there is a Radon measure in $\mathbb{R}$ which is absolutely continuous with respect to the $\sigma$-ideal of all $\sigma$-strongly porous sets and is not absolutely continuous with respect to the $\sigma$ ideal of all $\sigma$-porous sets. Our arguments also show that the answer to the natural extension of this question to general metric spaces remains negative. We then turn our attention to (upper) porosities of measures and strengthen the results of [4] on attainable values of porosities of measures. The proofs presented here are simpler and work also in (separable) metric spaces. The key to all our results is the decomposition theorem for $\sigma$-porous sets of [7], whose appropriate version can be found in Proposition 1.3 below.

1991 Mathematics Subject Classification. 28A05, 28A12.

Key words and phrases. Upper porosity, strongly porous sets, porosity of measures. 
Before giving a more detailed account of our results, we would like to make two remarks on the notion of porosity and its generalizations.

Quantitative notions of porosity of a subset $S$ of $\mathbb{R}^{n}$ at a point $x$ go back to Denjoy. They are defined by limit behaviour, as $r \rightarrow 0$, of the maximal ratio $s / r$, where $s$ are such that the ball $B(x, r)$ centered at $x$ with radius $r$ contains a ball $B(z, s)$ which does not meet $S$. Depending on applications, the limit behaviour is defined as either upper or lower limit, and both these (non-equivalent) notions are called "porosity of $S$ at $x "$. Since this inconsistency has caused some confusion, here we use the terms upper and lower porosity $S$ at $x$. However, since our results concern only the case of upper porosity, we speak about porous or $\sigma$-porous sets without mentioning the adjective "upper".

Note that "upper porosity" corresponds to the Denjoy-Dolzhenko notion of porosity. Also note that "lower porous sets" correspond to "very porous sets". See [8] for further information and results related to various notions of porosity.

Like many other authors, we are interested in porosity notions in metric spaces. The above definition is sometimes used, but in strange metric spaces it may exhibit unexpected behaviour (cf Remark 1.2(iv)). We correct this situation by requiring that the ball $B(z, s)$ is contained in $B(x, r)$ not only geometrically but also algebraically, i.e. that $\operatorname{dist}(z, x)+s \leq r$. One of the advantages of this (admittedly somewhat formal) concept is that it gives natural results when used to define the notion of porosity of measures. Had we been interested in porosity of sets only, we would probably base our notion on the concept of $\langle g\rangle$ porosity from $[7,8]$ : we would not require that $B(z, s) \subset B(x, r)$ but that $z \in B(x, r)$. However, this concept does not extend the above notion from $\mathbb{R}^{n}$, although the two concepts become equivalent after a simple rescaling (in fact, the new notion is rescaling equivalent to the notion we use; see Remark 1.2(iii)).

Definition 1.1. Let $(X, \rho)$ be a metric space. We denote by $B(x, r)$ the open ball with center $x \in X$ and radius $r$. For $A \subset X, x \in X$, and $r>0$ let

$$
\begin{array}{r}
\gamma(A, x, r):=\sup \{s>0: \text { there is } z \text { such that } \varrho(z, x)+s \leq r \\
\text { and } B(z, s) \cap A=\emptyset\}
\end{array}
$$

(where we put $\sup \emptyset:=0$ ). The upper porosity of $A$ at the point $x$ and the upper porosity of the set $A$ are defined by

$$
\bar{p}(A, x):=\limsup _{r \downarrow 0} \frac{\gamma(A, x, r)}{r} \text { and } \bar{p}(A):=\inf \{\bar{p}(A, x): x \in A\},
$$

respectively. 
We say that $A \subset X$ is porous if $\bar{p}(A, x)>0$ for each $x \in A$.

A set $A \subset \mathbb{R}$ is called strongly porous if $\bar{p}(A)=1 / 2$ or $A=\emptyset$.

We say that $A$ is $\sigma$-porous ( $\sigma$-strongly porous) if it is a countable union of porous (strongly porous) sets.

Remark 1.2. (i) It is easy to see that $\bar{p}(A, x)=1$ if $x \notin \bar{A}$ and $0 \leq \bar{p}(A, x) \leq 1 / 2$ in the opposite case. Therefore $\bar{p}(A) \leq 1 / 2$ for each nonempty $A \subset X$.

(ii) If $x \in \bar{A}$, then it is easy to see that $\bar{p}(A, x)=1 / 2$ iff there exists a sequence of balls $B\left(z_{n}, s_{n}\right)$ such that $z_{n} \rightarrow x, B\left(z_{n}, s_{n}\right) \cap A=\emptyset$ and $s_{n} / \rho\left(x, z_{n}\right) \rightarrow 1$.

(iii) The notion of porosity stemming from the $\langle g\rangle$-porosity of $[7,8]$ (cf also [9]) would replace $\gamma(A, x, r)$ by $\gamma\langle A, x, r\rangle:=\sup \{s$ : $B(z, s) \cap A=\emptyset$ for some $z \in B(x, r)\}$ and define $\bar{p}\langle A, x\rangle:=$ $\lim \sup _{r \downarrow 0} \frac{\gamma\langle A, x, r\rangle}{r}$. If $x \in \bar{A}$ then clearly $\bar{p}\langle A, x\rangle=\bar{p}(A, x) /(1-$ $\bar{p}(A, x))$ and $\bar{p}(A, x)=\bar{p}\langle A, x\rangle /(1+\bar{p}\langle A, x\rangle)$, hence this notion is rescaling equivalent to that of Definition 1.1.

(iv) As was already mentioned, in general metric spaces the geometric condition $B(z, s) \subset B(x, r)$ is less natural than the algebraic one $\rho(z, x)+s \leq r$.

To see this, put

$$
\begin{gathered}
S:=\bigcup_{n=0}^{\infty}\left[4^{-2 n-1}, 4^{-2 n}\right], T:=\bigcup_{n=0}^{\infty}\left\{4^{-2 n-1}\right\}, \\
X:=S \cup(-S) \cup\{0\}, A:=T \cup(-T) \cup\{0\} .
\end{gathered}
$$

It is easy to see that in the metric space $X$ (with the Euclidean metric) the set $A$ is not strongly porous ( since $\bar{p}(A, 0)=3 / 7<$ $1 / 2)$ but with the geometrical definition the upper porosity of $A$ would equal $3 / 4>1 / 2$.

This example confirms the observation made on several occasions that the geometrical definition of strong porosity in general metric spaces (proposed, for example, in [8]) should be replaced by a more restrictive one.

The key to our results is the following statement which can be easily deduced from Proposition 4.1 of [7] (Proposition 4.4 of [8]). In these references, the statement is based on the notion of porosity indicated in Remark 1.2(iii)), which is the most natural porosity notion in this context. The reader may therefore find our adaptation of the proof somewhat artificial. 
Proposition 1.3. Let $X$ be a metric space, $A \subset X$ be a $\sigma$-porous set and $p<1 / 2$. Then $A$ can be written as $A=\bigcup_{n=1}^{\infty} A_{n}$ where $\bar{p}\left(A_{n}\right)>p$ for each $n$.

Proof. It is clearly sufficient to prove the assertion in the case when $\bar{p}(A, x)>0$ for each $x \in A$. Choose $0<q<1$ such that $q /(1+q)>p$. For $k, l=1, \ldots$ define $A_{k, l}:=\left(\cap_{m=1}^{\infty} M_{k, m}\right) \backslash M_{k-1, l}$, where $M_{0, l}=\emptyset$ and, for $k>0, M_{k, l}$ is the union of all balls $B(z, r)$ where $0<r<1 / l$ and $B\left(z, q^{k} r\right) \cap A=\emptyset$.

Whenever $x \in A_{k, l}$ and $m \geq l$, we use that $x \in M_{k, m}$ to find $0<r<$ $1 / m$ and $z \in X$ such that $x \in B(z, r)$ and $B\left(z, q^{k} r\right) \cap A=\emptyset$. Since $B\left(z, q^{k-1}(q r)\right)=B\left(z, q^{k} r\right)$, we have $B(z, q r) \subset M_{k-1, l} \subset X \backslash A_{k, l}$ and $\varrho(z, x)+q r \leq(1+q) r$, hence $\gamma\left(A_{k, l}, x,(1+q) r\right) \geq q r$. Since $m \geq l$ was arbitrary, we conclude that $\bar{p}\left(A_{k, l}, x\right) \geq q /(1+q)>p$.

To finish the proof, it is enough to show that $A \subseteq \bigcup_{k=1}^{\infty} \bigcup_{l=1}^{\infty} A_{k, l}$. Let $x \in A$ and find $k$ so that $\bar{p}(A, x)>q^{k}$. Then for each $m$ there are $0<r<1 / m, s>q^{k} r$ and $z \in X$ such that $\varrho(z, x)+s \leq r$ and $B(z, s) \cap A=\emptyset$. Hence $x \in B(z, r)$ and $B\left(z, q^{k} r\right) \cap A=\emptyset$, which shows that $x \in M_{k, m}$. It follows that each $x \in A$ belongs to $\bigcap_{m=1}^{\infty} M_{k, m}$ for some $k \geq 1$; taking the least $k$ with this property we have that $x \notin M_{k-1, l}$ for some $l$, hence $x \in A_{k, l}$, as required.

We will need also the following simple fact.

Lemma 1.4. For every subset $A$ of a metric space $X$ there is a Borel set $B \supset A$ such that $\bar{p}(B)=\bar{p}(A)$.

Proof. We may assume that $p:=\bar{p}(A)>0$ (otherwise consider $B=X$ ). For $k, l=1, \ldots$ denote by $G_{k, l}$ the set of those $x$ for which there are $0<r<1 / l, s>(\bar{p}(A)-1 / k) r$ and $z \in X$ such that $\varrho(z, x)+s \leq r$ and $B(z, s) \cap A=\emptyset$. Then each $G_{k, l}$ is open and $B:=\bigcap_{k, l=1}^{\infty} G_{k, l}$ is a $G_{\delta}$ set such that $A \subset B$ and $\bar{p}(B)=\bar{p}(A)$.

\section{Measures absolutely continuous with Respect to POROUS AND STRONGLY POROUS SETS}

Let $X$ be a metric space. We will say that $\mu$ is a Borel regular measure on $X$ if it is a complete measure for which all Borel sets are measurable and

$$
\mu(M)=\sup \{\mu(F): F \subset M, F \text { is closed }\}
$$

for each $\mu$-measurable set $M$.

The full strength of Proposition 1.3 will now be used to show the basic fact that $\sigma$-porous sets of finite measure differ from strongly porous sets by sets of arbitrarily small measure. 
Proposition 2.1. Let $\mu$ be a Borel regular measure on a metric space $X$ and let $A \subset X$ be a $\mu$-measurable $\sigma$-porous set of finite measure. Then for every $\varepsilon>0$ there is a closed strongly porous set $S$ such that $\mu(A \backslash S)<\varepsilon$.

Proof. Fix for a moment a natural number $n$ and use Proposition 1.3 to find sets $A_{k}^{n}$ such that $\bar{p}\left(A_{k}^{n}\right)>\frac{1}{2}-\frac{1}{n}$ and $\bigcup_{k=1}^{\infty} A_{k}^{n}=A$. By Lemma 1.4 there are Borel sets $B_{k}^{n} \supset A_{k}^{n}$ with $\bar{p}\left(B_{k}^{n}\right)=\bar{p}\left(A_{k}^{n}\right)$. The sets $C_{k}^{n}:=B_{k}^{n} \cap A$ are $\mu$-measurable and we can find an index $k_{n}$ such that $\mu\left(\bigcup_{k=1}^{k_{n}} C_{k}^{n}\right)>\mu(A)-2^{-n} \varepsilon$. Since $\mu$ is regular, we can choose closed sets

$$
F_{1}^{n} \subset C_{1}^{n}, F_{2}^{n} \subset C_{2}^{n} \backslash C_{1}^{n}, \ldots, F_{k_{n}}^{n} \subset C_{k_{n}}^{n} \backslash\left(C_{k_{n}-1}^{n} \cup \cdots \cup C_{1}^{n}\right)
$$

such that $\mu\left(\bigcup_{k=1}^{k_{n}} F_{k}^{n}\right)>\mu(A)-2^{-n} \varepsilon$. Put $S_{n}:=\bigcup_{k=1}^{k_{n}} F_{k}^{n}$. Since any point of $F_{k}^{n}, k=1, \ldots, k_{n}$ has a positive distance from the set $S_{n} \backslash F_{k}^{n}$, it is easy to see that $S_{n}$ is a closed set satisfying $\bar{p}\left(S_{n}\right)>$ $\frac{1}{2}-\frac{1}{n}$. Consequently $S:=\bigcap_{n=1}^{\infty} S_{n}$ is a closed strongly porous set. Since $A \backslash S \subset \bigcup_{n=1}^{\infty}\left(A \backslash S_{n}\right)$, we obtain $\mu(A \backslash S)<\varepsilon$.

We say that $\mu$ is absolutely continuous with respect to a $\sigma$-ideal $I$ if $\mu(A)=0$ for each $A \in I$. Note that Tkadlec [6] proved that there is a singular Radon measure on $\mathbb{R}$ which is absolutely continuous with respect to the $\sigma$-ideal of all $\sigma$-porous sets.

Theorem 2.2. Let $X$ be a metric space and let $\mu$ be a $\sigma$-finite Borel regular measure on $X$. Then the following assertions hold.

(i) If $A \subset X$ is $\sigma$-porous and $\mu$-measurable, then $\mu(A)=\sup \{\mu(S): S \subset A, S$ is closed and strongly porous $\}$.

(ii) If $B \subset X$ is $\sigma$-porous and $\mu$-measurable, then there exists an $F_{\sigma} \sigma$-strongly porous set $K \subset B$ such that $\mu(B \backslash K)=0$.

(iii) For every $\sigma$-porous set $C \subset X$ there exists a $\sigma$-strongly porous set $T \subset C$ such that $\mu(C \backslash T)=0$.

(iv) If $\mu$ is absolutely continuous with respect to the $\sigma$-ideal of all $\sigma$ strongly porous sets, then it is absolutely continuous with respect to the $\sigma$-ideal of all $\sigma$-porous sets.

Proof. The statement (i) is obtained by writing $A$ as an increasing union of $\mu$-measurable sets $A_{n}$ of finite measure and using Proposition 2.1 for each $n$.

To prove (ii), write $B$ as an increasing union of $\mu$-measurable sets $B_{n}$ of finite measure, use Proposition 2.1 to find closed strongly porous sets $K_{n} \subset B_{n}$ such that $\mu\left(B_{n} \backslash K_{n}\right)<2^{-n}$ and put $K:=\bigcup_{n=1}^{\infty} K_{n}$. 
To prove (iii), find by Proposition 1.3 and Lemma 1.4 a $\sigma$-porous Borel set $B \supset C$ and then find a $\sigma$-strongly porous $F_{\sigma}$ set $K \subset B$ as in (ii). It is clearly sufficient to put $T:=C \cap K$.

The assertion (iv) immediately follows from (iii).

\section{UPPER POROSITIES OF MEASURES}

Let $\mu$ be a Borel regular probability measure on a separable metric space $X$. Some possible definitions of the notion of a lower porosity of $\mu$ were investigated in [1]. Analogous definitions of an upper porosity of $\mu$ were studied in [4]. For our investigations, the key notion is given in the following definition.

Definition 3.1. We denote

$$
\begin{gathered}
\gamma(\mu, x, r, \varepsilon):=\sup \{s>0: \text { there is } z \text { such that } \varrho(x, z)+s \leq r \\
\text { and } \mu(B(z, s)) \leq \varepsilon \mu(B(x, r))\}
\end{gathered}
$$

and we define the upper porosity of the measure $\mu$ at a point $x$ by

$$
\overline{\operatorname{por}}(\mu, x):=\lim _{\varepsilon \downarrow 0} \limsup _{r \downarrow 0} \frac{\gamma(\mu, x, r, \varepsilon)}{r} .
$$

Note that $0 \leq \overline{\operatorname{por}}(\mu, x) \leq 1$ for all $x$. (Recall that $\sup \emptyset=0$.)

The following simple fact will be used in the sequel without any special reference.

Proposition 3.2. The function $x \rightarrow \overline{\mathrm{por}}(\mu, x)$ is Borel measurable.

Proof. Since the functions $\varepsilon \rightarrow \gamma(\mu, x, r, \varepsilon)$ are clearly non-decreasing on $(0, \infty)$,

$$
\overline{\operatorname{por}}(\mu, x):=\lim _{k \rightarrow \infty} \lim _{l \rightarrow \infty} \sup \left\{\frac{\gamma(\mu, x, r, 1 / k)}{r}: 0<r<1 / l\right\} .
$$

Thus it suffices to check that for each fixed $k, l \in \mathbb{N}$ the function $x \rightarrow$ $\sup \left\{\frac{\gamma(\mu, x, r, 1 / k)}{r}: 0<r<1 / l\right\}$ is lower semi-continuous, which is an easy task.

Recall that $\mu$ is said to satisfy the doubling condition at $x$ if there are $r_{0}>0$ and $c<\infty$ such that $\mu(B(x, 2 r)) \leq c \mu(B(x, r))$ whenever $0<r<r_{0}$. This may be equivalently defined by replacing 2 by any number greater than one. Another equivalent form is obtained by fixing an arbitrary $0<\eta<1$ and requiring that there are $r_{0}>0$ and $\varepsilon>0$ such that $\mu(B(x, \eta r)) \geq \varepsilon \mu(B(x, r))$ for $0<r<r_{0}$.

Note that by the above definitions the doubling condition is satisfied at every point not belonging to the support of $\mu$ and $\overline{\operatorname{por}}(\mu, x)=1$ at such points. 
Proposition 3.3. Let $x$ be in the support of $\mu$. Then the following statements are equivalent

(i) $\overline{\operatorname{por}}(\mu, x)>1 / 2$,

(ii) $\overline{\operatorname{por}}(\mu, x)=1$,

(iii) $\mu$ does not satisfy the doubling condition at $x$.

Proof. Suppose that $\overline{\operatorname{por}}(\mu, x)>1 / 2$ and find an $\eta>0$ such that $\overline{\operatorname{por}}(\mu, x)>\eta+1 / 2$. Then for every $0<\varepsilon<1$ there are an arbitrarily small $r>0, z \in X$ and $s>(\eta+1 / 2) r$ such that $\varrho(x, z)+s \leq$ $r$ and $\mu(B(z, s)) \leq \frac{\varepsilon}{2} \mu(B(x, r))$. Since the above inequalities imply $\varrho(x, z)<r / 2-\eta r$, we obtain $B(x, \eta r) \subset B(z, s)$, and consequently $0<\mu(B(x, \eta r)) \leq \frac{\varepsilon}{2} \mu(B(x, r))<\varepsilon \mu(B(x, r))$. Hence $\mu$ does not satisfy the doubling condition at $x$.

Suppose now that $\mu$ does not satisfy the doubling condition at $x$ and fix $0<\eta<1$. Then for every $\varepsilon>0$ there are arbitrarily small $r>0$ such that $\mu(B(x, \eta r))<\varepsilon \mu(B(x, r))$. For these $r$ we get (with $z=x$ ) that $\gamma(\mu, x, r, \varepsilon) \geq \eta r$, which shows that $\overline{\operatorname{por}}(\mu, x) \geq \eta$. Since $0<\eta<1$ was arbitrary, we have $\overline{\operatorname{por}}(\mu, x)=1$.

Proposition 3.4. For every $\varepsilon>0$ there is a closed strongly porous subset $S$ of the set $P:=\{x: \overline{\operatorname{por}}(\mu, x)>0\}$ such that $\mu(P \backslash S)<\varepsilon$.

Proof. Find $p>0$ such that $\mu\{x \in P: \overline{\operatorname{por}}(\mu, x) \leq 2 p\}<\varepsilon / 2$ and denote $P_{0}:=\{x: \overline{\operatorname{por}}(\mu, x)>2 p\}$. Let $\eta_{j}>0$ be such that $\sum_{j=1}^{\infty} \eta_{j}<$ $\varepsilon / 4$.

We note that for each $x \in P_{0}, \eta>0$ and $\delta>0$ there is a $0<r<\delta / 2$ such that, for some $z \in X$ we have $\varrho(z, x)+2 p r \leq r$ and $\mu(B(z, 2 p r)) \leq$ $\eta \mu(B(x, r))$. Letting $s=2 r$, we infer that $P_{0}$ is covered by the family

$$
\{B(z, s): s<\delta \text { and } \mu(B(z, p s)) \leq \eta \mu(B(z, s))\} .
$$

Hence for each $j=1,2, \ldots$ we may use Theorem 2.8.4 from [2] to find a disjoint family $B\left(z_{i}, s_{i}\right)\left(i \in I_{j}\right)$ such that $P_{0} \subset \bigcup_{i \in I_{j}} B\left(z_{i}, 5 s_{i}\right)$, and $s_{i}<1 / j$ and $\mu\left(B\left(z_{i}, p s_{i}\right)\right) \leq \eta_{j} \mu\left(B\left(z_{i}, s_{i}\right)\right)$ for each $i \in I_{j}$. Denote $G_{j}:=\bigcup_{i \in I_{j}} B\left(z_{i}, p s_{i}\right)$. Since $B\left(z_{i}, s_{i}\right),\left(i \in I_{j}\right)$ are disjoint balls, the set $I_{j}$ is countable and

$$
\mu\left(G_{j}\right)=\sum_{i \in I_{j}} \mu\left(B\left(z_{i}, p s_{i}\right)\right) \leq \eta_{j} \sum_{i \in I_{j}} \mu\left(B\left(z_{i}, s_{i}\right)\right) \leq \eta_{j} .
$$

Let $G=\bigcup_{j=1}^{\infty} G_{j}$. Then $\mu(G) \leq \sum_{j=1}^{\infty} \eta_{j}<\varepsilon / 4$ and, since $1 / j \rightarrow 0$, $\bar{p}\left(P_{0} \backslash G\right) \geq p /(5+p)$. Hence by Theorem $2.2(\mathrm{i})$ there is a closed strongly porous subset $S$ of $P_{0}$ such that $\mu\left(\left(P_{0} \backslash G\right) \backslash S\right)<\varepsilon / 4$. Clearly, $S$ has the required properties. 
Proposition 3.5. Every $\sigma$-porous subset of the set $\{x: \overline{\operatorname{por}}(\mu, x)<$ $1 / 2\}$ has $\mu$ measure zero.

Proof. If $\overline{\operatorname{por}}(\mu, x)<1 / 2$, then $x$ belongs to the support of $\mu$ and thus by Proposition 3.3 there exist $k, l \in \mathbb{N}$ such that

$$
\mu(B(x, r)) \geq \frac{1}{k} \mu(B(x, 5 r)) \text { for } r<\frac{1}{l} .
$$

Let $n \in \mathbb{N}$ be such that $\overline{\operatorname{por}}(\mu, x)<1 / 2-1 / n$ and find $p \in \mathbb{N}$ and $q \in \mathbb{N}$ such that

$$
\frac{1}{r} \gamma(\mu, x, r, 1 / p)<\frac{1}{2}-\frac{1}{n} \text { for each } r<\frac{1}{q} .
$$

Thus, denoting by $A(k, l, n, p, q)$ the set of those $x \in X$ for which (3.1) and (3.2) hold, we have

$$
A:=\{x: \overline{\operatorname{por}}(\mu, x)<1 / 2\} \subset \bigcup\{A(k, l, n, p, q): k, l, n, p, q \in \mathbb{N}\} .
$$

Now suppose that a $\sigma$-porous set $P \subset A$ is given. By Theorem 2.2(iii) there is a $\sigma$-strongly porous set $Q \subset P$ with $\mu(P \backslash Q)=0$. Since each set $Q \cap A(k, l, n, p, q)$ is $\sigma$-strongly porous, it is sufficient to prove that, for arbitrary fixed $k, l, n, p, q$, each strongly porous subset $S$ of $A(k, l, n, p, q)$ is $\mu$-null. Suppose to the contrary that $\mu^{*}(S)>0$, where $\mu^{*}$ is the outer measure determined by $\mu$. Choose an open set $G \supset S$ such that $\mu(G)<\mu^{*}(S)\left(1+\frac{1}{p k}\right)$. Since $S$ is strongly porous, we can assign to each $x \in S$ numbers $0<r_{x}<\min (1 / l, 1 / q), 0<s_{x}$ and a point $z_{x} \in X$ such that

$$
B\left(x, r_{x}\right) \subset G, \rho\left(x, z_{x}\right)+s_{x} \leq r_{x}, B\left(z_{x}, s_{x}\right) \cap S=\emptyset \text { and } \frac{s_{x}}{r_{x}}>\frac{1}{2}-\frac{1}{n} .
$$

By (3.2) we then obtain

$$
\mu\left(B\left(z_{x}, s_{x}\right)\right)>\frac{1}{p} \mu\left(B\left(x, r_{x}\right)\right) .
$$

Using Theorem 2.8.4 from [2] there is a subset $\left\{x_{i}: i \in I\right\}$ of $S$ such that the balls $B\left(x_{i}, r_{x_{i}}\right)$ are disjoint and $S \subset \bigcup_{i \in I} B\left(x_{i}, 5 r_{x_{i}}\right)$; clearly $I$ is countable. Denoting $D:=\bigcup_{i \in I} B\left(z_{x_{i}}, s_{x_{i}}\right)$, we have

$$
\begin{aligned}
\mu D & =\sum_{i \in I} \mu\left(B\left(z_{x_{i}}, s_{x_{i}}\right)\right) \geq \frac{1}{p} \sum_{i \in I} \mu\left(B\left(x_{i}, r_{x_{i}}\right)\right) \\
& \geq \frac{1}{p k} \sum_{i \in I} \mu\left(B\left(x_{i}, 5 r_{x_{i}}\right)\right) \geq \frac{1}{p k} \mu^{*}(S)
\end{aligned}
$$

and therefore, since $D$ is a $\mu$-measurable subset of $G$ and $D \cap S=\emptyset$, we obtain $\mu G \geq\left(1+\frac{1}{p k}\right) \mu^{*}(S)$, which is a contradiction. 
We now summarize the previous results on porosity of measures into the following theorem.

Theorem 3.6. Let $\mu$ be a Borel regular probability measure on a separable metric space $X$ and let $S_{p}:=\{x: \overline{\operatorname{por}}(\mu, x)=p\}$. Then the following statements hold.

(i) $S_{p}=\emptyset$ for each $1 / 2<p<1$.

(ii) $S_{1}=N \cup(X \backslash \operatorname{spt}(\mu))$ where $N$ is the set of those $x$ at which $\mu$ does not satisfy the doubling condition.

(iii) $\mu\left(X \backslash\left(S_{0} \cup S_{1 / 2} \cup S_{1}\right)\right)=0$.

(iv) There exists a $\sigma$-strongly porous set $A \subset S_{1 / 2} \cup S_{1}$ such that $\mu\left(\left(S_{1 / 2} \cup S_{1}\right) \backslash A\right)=0$.

(v) Every $\sigma$-porous subset of $S_{0}$ has $\mu$ measure zero.

Proof. The statements (i) and (ii) follow from Proposition 3.3. Using (i), we have that $B:=X \backslash\left(S_{0} \cup S_{1 / 2} \cup S_{1}\right)=\{x: 0<\overline{\operatorname{por}}(\mu, x)<$ $1 / 2\}$. Proposition 3.4 implies that there is a $\sigma$-porous set $P \subset B$ such that $\mu(B \backslash P)=0$. By Proposition 3.5 we have $\mu(P)=0$, hence $\mu(B)=0$, which is (iii). The statements (iv) and (v) follow directly from Propositions 3.4 and 3.5, respectively.

This Theorem immediately implies a generalization, with a simple proof, of the results of [4] on attainable values of the following porosities of measures:

$$
\begin{aligned}
\overline{\operatorname{por}}(\mu) & :=\inf \{s: \overline{\operatorname{por}}(\mu, x) \leq s, \text { for } \mu \text {-a.e. } x \in X\}, \\
\bar{p}(\mu) & :=\sup \{\bar{p}(A): A \subset X \text { with } \mu(A)>0\}, \\
\bar{p}^{*}(\mu) & :=\inf _{\delta>0} \sup \{\bar{p}(A): A \subset X \text { with } \mu(A)>1-\delta\},
\end{aligned}
$$

and

$$
\bar{p}^{* *}(\mu):=\sup \{\bar{p}(A): A \subset X \text { with } \mu(A)=1\} .
$$

The following statement easily follows from Theorem 3.6.

Theorem 3.7. Let $\mu$ be a Borel regular probability measure on a separable metric space $X$. Then $\overline{\operatorname{por}}(\mu)$ can attain only the values $0, \frac{1}{2}$ and 1 , and each of $\bar{p}(\mu)$ and $\bar{p}^{*}(\mu)$ can attain only the values 0 and $\frac{1}{2}$.

Moreover,

$$
0 \leq \bar{p}(\operatorname{spt}(\mu)) \leq \bar{p}^{* *}(\mu) \leq \bar{p}^{*}(\mu) \leq \bar{p}(\mu) \leq \overline{\operatorname{por}}(\mu) \leq 1 .
$$

If $\mu$ satisfies the doubling condition $\mu$-a.e., then $\overline{\operatorname{por}}(\mu)=\bar{p}(\mu) \leq \frac{1}{2}$, in the opposite case $\bar{p}(\mu)=\frac{1}{2}$ and $\overline{\operatorname{por}}(\mu)=1$.

The following result on porosity decomposition of measures is an immediate consequence of Theorem 3.6. We also give a simple independent proof whose (minor) advantage is that it works directly in 
non-separable spaces and can therefore be used to deduce some of the statements of Theorem 3.7 for non-separable spaces. Note however, that some of these statements may fail: see Remark 4.7 or observe that the support of $\mu$ may be undefined.

Proposition 3.8. Let $\mu$ be a Borel regular probability measure on a metric space $X$. Then there is a $\sigma$-strongly porous Borel set $S \subset X$ such that every $\sigma$-porous subset of $X \backslash S$ has $\mu$ measure zero.

Proof. Put

$$
\alpha:=\sup \{\mu(S): S \text { is a } \sigma-\text { strongly porous set }\} .
$$

For each natural number $n$ use Lemma 1.4 to choose a Borel $\sigma$-strongly porous set $S_{n}$ with $\mu\left(S_{n}\right)>\alpha-1 / n$. Then $S:=\bigcup_{n=1}^{\infty} S_{n}$ is a Borel $\sigma$-strongly porous set and $\mu(S)=\alpha$. To prove our assertion consider a $\sigma$-porous set $P \subset X \backslash S$. By Theorem 2.2(iii) find a $\sigma$-strongly porous set $T \subset P$ such that $\mu(P \backslash T)=0$. Then $\alpha \geq \mu(S \cup T)=\mu(S)+\mu(T)=$ $\alpha+\mu(T)$, so $\mu(P)=\mu(T)=0$.

Remark 3.9. Note that in the case $\mu(S)=0$ we have that $\mu$ is absolutely continuous with respect to $\sigma$-porous sets and $\bar{p}^{*}(\mu)=\bar{p}(\mu)=0$, in the case $0<\mu(S)<1$ we have that $\bar{p}^{*}(\mu)=0$ and $\bar{p}(\mu)=1 / 2$ and in the case $\mu(S)=1$ we have that $\bar{p}^{*}(\mu)=\bar{p}(\mu)=1 / 2$.

\section{EXAMPLES}

We finish with several easy examples. We first show that all the alternatives from Remark 3.9 may occur; in particular the inequality $\bar{p}^{*}(\mu) \leq \bar{p}(\mu)$ can be strict.

Example 4.1. Let $\lambda$ be the Lebesgue measure on $[0,1]$ and let $\nu$ be the probability measure on $\mathbb{R}$ given by $\nu\{0\}=1$. The case $\mu(S)=0$ of Remark 3.9 holds for $\mu=\lambda$, the case $0<\mu(S)<1$ holds for $\mu=\frac{\lambda+\nu}{2}$ and the case $\mu(S)=1$ holds for $\mu=\nu$.

Examples 4.2 and 4.3 show that the inequalities $\bar{p}^{* *}(\mu) \leq \bar{p}^{*}(\mu)$ and $\bar{p}(\operatorname{spt}(\mu)) \leq \bar{p}^{* *}(\mu)$ can be strict.

Example 4.2. Let $\mu$ be the probability measure on $\mathbb{R}$ given by $\mu\left\{\frac{1}{k}\right\}=$ $\mu\left\{-\frac{1}{k}\right\}=2^{-(k+2)}$ and $\mu\{0\}=2^{-1}$. The set $S=\{0\} \cup\{ \pm 1 / k: k=$ $1,2, \ldots\}$ does not have any proper subset with full $\mu$-measure. Since $\bar{p}(S, 0)=0$ we get $\bar{p}^{* *}(\mu)=0$. The set $S_{k}=S \backslash\left(0, \frac{1}{k}\right]$ satisfies $\mu\left(S_{k}\right)=$ $1-2^{-(k+1)}$ and $\bar{p}\left(S_{k}\right)=\frac{1}{2}$. Thus $\bar{p}^{*}(\mu)=\frac{1}{2}$.

Example 4.3. Let $\mu$ be the probability measure on $\mathbb{R}$ given by $\mu\left\{\frac{1}{k}\right\}=$ $\mu\left\{-\frac{1}{k}\right\}=2^{-k-1}$. Then $\bar{p}(\operatorname{spt}(\mu))=0$ and $\bar{p}^{* *}(\mu)=\frac{1}{2}$. 
Finally, the following example shows that $\bar{p}^{* *}(\mu)$ (on $\mathbb{R}$ ) does not have a dichotomic behaviour.

Example 4.4. Let $a>1$ and let $\mu$ be the probability measure on $\mathbb{R}$ given by $\mu\{0\}=2^{-1}$ and $\mu\left\{a^{-k}\right\}=\mu\left\{-a^{-k}\right\}=2^{-(k+2)}$. It is easy to see that the set $S=\{0\} \cup\left\{ \pm a^{-k}: k=1,2, \ldots\right\}$ satisfies $\bar{p}(S, 0)=\frac{a-1}{2 a}$. Since $S$ does not have any proper subset of full $\mu$-measure we get that $\bar{p}^{* *}(\mu)=\bar{p}(S)=\bar{p}(S, 0)=\frac{a-1}{2 a}$.

The following example shows that each of the sets $S_{0}, S_{1 / 2}, S_{1}$ of Theorem 3.6 may have positive measure.

Example 4.5. Let $\nu$ be the probability measure on $\mathbb{R}$ given by $\nu\{0\}=$ $1, \omega$ a probability measure on $[2,3]$ such that the set $N$ of those $x \in$ $[2,3]$ at which $\omega$ does not satisfy the doubling condition has full $\omega$ measure (see, for example, $\left[1\right.$, Example 4]) and let $\mu=\frac{\nu+\omega+\lambda}{3}$, where $\lambda$ is as in Example 4.1. Then $S_{0} \supset(0,1), S_{1 / 2} \supset\{0,1\}$ and $S_{1} \supset N$, so all these sets have positive $\mu$ measure.

Our next example points out that the key Proposition 2.1 does not hold without the finiteness assumption.

Example 4.6. Whenever $A$ is a countable subset of a metric space $X$ which is porous but not strongly porous (for example the set $A$ and metric space $X$ from Remark 1.2(iv)), and $\mu(E)$ is the number of elements of $E \cap A$, then $\mu$ is a $\sigma$-finite Borel regular measure on $X$ for which there is no strongly porous set $S$ with $\mu(A \backslash S)<1$.

Remark 4.7. The separability of the space $X$ in Section 3 may be replaced by requiring, for example, that $\mu$ be a Radon measure but cannot be completely removed. To illustrate this, assume existence of a measurable cardinal and consider a discrete metric space $X$ on which there is a probability measure $\mu$ defined on all subsets such that singletons have measure zero. Then $\overline{\operatorname{por}}(\mu, x)=1$ for all $x \in X$ but the statement of Proposition 3.4 fails since $X$ contains no non-empty strongly porous subset. Moreover, $\bar{p}(\mu)=0$ and so the last statement of Theorem 3.7 fails as well.

Remark 4.8. We should also point out that our results have no analogy for lower porosity. Indeed, for this it suffices to take any measure on $\mathbb{R}$ supported by a lower porous set such that every set of positive measure has positive Hausdorff dimension. (For example, consider the Cantor measure.) Then there is no strongly lower porous set of positive measure since every strongly lower porous subset of $\mathbb{R}$ has Hausdorff dimension zero. (See [5] or, for analogous results concerning porosities of measures, [3].) 
Acknowledgment. The research of the first and second authors (M. E. Mera and M. Morán) was partially supported by the grant BXX2000 - 0639 from the Ministry of Science and Technology. The research of the fourth author (L. Zajíček) was partially supported by the grant GAČR 201/00/0767 from the Grant Agency of Czech Republic, by the grant GAUK 160/1999 from Charles University, Prague, and by the grant MSM 113200007 from the Czech Ministry of Education.

\section{REFERENCES}

[1] J-P. Eckmann, E. Järvenpää and M. Järvenpää, Porosities and Dimensions of Measures, Nonlinearity 13 (2000), 1-18.

[2] H. Federer, Geometric measure theory, Die Grundlehren der mathematischen Wissenschaften, Band 153, Springer-Verlag, New York 1969.

[3] E. Järvenpää and M. Järvenpää, Porous measures in $R^{n}$ : Local structure and dimensional properties, Proc. Amer. Math. Soc. 130(2002), 419-426.

[4] M. E. Mera and M. Morán, Attainable values for upper porosities of measures, Real Anal. Exchange 26 (2000/2001), 101-115.

[5] A. Salli, On the Minkowski dimension of strongly porous fractal sets in $R^{n}$, Proc. London Math. Soc. (3) 62 (1991), no. 2, 353-372.

[6] J. Tkadlec, Construction of a finite Borel measure with $\sigma$-porous sets as null sets, Real Anal. Exchange 12 (1986/87), 349-353.

[7] L. Zajíček, Sets of $\sigma$-porosity and sets of $\sigma$-porosity $(q)$, Casopis Pěst. Mat. 101 (1976), 350-359.

[8] L. Zajíček, Porosity and $\sigma$-porosity, Real Anal. Exchange 13 (1987/88), 314-350.

[9] M. Zelený and J. Pelant, The structure of the $\sigma$-ideal of $\sigma$-porous sets, (submitted), available electronically under http://www.karlin.mff.cuni.cz/kmapreprints/.

Departamento de Análisis Económico I, Universidad Complutense, Campus de Somosaguas 28223, Madrid, Spain

E-mail address: ececo05@sis.ucm.es

Departamento de Análisis Económico I, Universidad Complutense, Campus de Somosaguas 28223, Madrid, Spain

E-mail address: ececo06@sis.ucm.es

Department of Mathematics, University College London, Gower Street, London WC1E 6BT, United Kingdom

E-mail address: dp@math.ucl.ac.uk

Department of Mathematical Analysis, Charles University, Sokolovská 83, 18600 Prague 8, Czech Republic

E-mail address: zajicek@karlin.mff.cuni.cz 